\title{
Adenocarcinoma primario en un remanente ureteral tras una nefrectomia simple
}

\author{
Juan P. Caballero Romeu, Juan A. Galán Llopis, Juan F. Galiano Baena, Adel Abuomar*, \\ Antonio M. Pelluch Auladell, Juan J. Lobato Encinas \\ Servicio de Urología. *Servicio de Anatomía Patológica. Hospital General Universitario de Alicante. España.
}

\begin{abstract}
Resumen
Objetivos: El adenocarcinoma primario de uréter representa un porcentaje mínimo de las neoplasias del tracto urinario superior. El escaso número de casos publicados no permite establecer unas pautas claras en su manejo clínico, diagnóstico y terapéutico. Nuestro objetivo es proponer una pauta de actuación que permita mejorar el pronóstico de esta patología.

Métodos: Presentamos un caso de adenocarcinoma primario de uréter que afecta a un remanente ureteral tras una nefrectomía simple y hacemos una revisión de la literatura al respecto.

Resultados: El paciente fue diagnosticado de adenocarcinoma primario de uréter tras el examen anatomopatológico y un estudio minucioso de extensión que permitiera descartar un tumor primario.

Conclusión: El adenocarcinoma primario de uréter es una entidad muy poco frecuente lo que condiciona una pobre caracterización clínica y anatomopatológica. Puede presentarse como un dolor en flanco, disuria urgencia y/o hematuria macroscópica en pacientes con antecedentes de nefrectomía. Ante el hallazgo de un adenocarcinoma de uréter debemos descartar siempre que se trate de un tumor metastásico. En cuanto al tratamiento parece necesaria la exéresis completa del tumor y se puede intentar el tratamiento quimioterápico adyuvante con resultados inciertos.
\end{abstract}

Palabras Clave: Adenocarcinoma, Litiasis, Metaplasia, Nefrectomía, Neoplasias Ureterales.

\section{Primary adenocarcinoma of a ureteral remnant stump after nephrectomy}

\section{Abstract}

Aim: Adenocarcinomas of the upper urinary tract are very rare malignancies often related to primary genitourinary and extraurinary tumours. Primary adenocarcinomas are even rarer. There are no clinical guidelines for the management of primary ureteral adenocarcinoma. Our objective is to propose a management algoritm of this sort of tumours.

Methods: We report a case of adenocarcinoma affecting a ureteral stump in a patient who previously underwent a nephrectomy. A review of literature is performed.

Results: After the ureterectomy the pathologist diagnosed a ureteral adenocarcinoma. We performed an extension study in order to discard an extraurinary origin.

Conclusions: Adenocarcinoma of the ureteral stump is a very rare malignancy. About the clinic and pathologic characterization, there is no an established reference due to the short number of previous reports. Clinical presentation may include previous nephrectomy, flank pain, dysuria, urgency and/or gross hematuria. It seem necessary the complete resection of the urinary tract when a nephrectomy is performed. Adjuvant chemotherapy can be employed with uncertain results.

Keywords: Adenocarcinoma, Lithiasis, Metaplasia, Nephrectomy, Ureteral Neoplasms.

$\mathrm{E}$ adenocarcinoma del tracto urinario es un tumor muy poco habitual y a menudo relacionado con tumores primarios con asiento en otro punto del tracto genitourinario o fuera de este. El adenocarcinoma primario del tracto superior es aún menos frecuente. Son tumores que tienen mal pronóstico. Los datos que nos pueden hacer sospechar una enfermedad originada en un remanente ureteral son una nefrectomía previa asociada a síndrome miccional, fiebre y/o dolor en flanco.

\section{CASO CLÍnico}

Un varón de 52 años acude a nuestro centro con fiebre, dolor en flanco derecho, polaquiuria y dispepsia gástrica. Desde hace dos años el paciente presenta ocasionalmente esta sintomatología desde que se le realizó una nefrectomía simple por una pielonefritis crónica sintomática. No tenía otros antecedentes de interés. El paciente presentaba una hidronefrosis derecha secundaria a una litiasis en la unión pieloureteral. Antes de la nefrectomía se le 
practicó una nefrostomía de descarga y posteriormente una Urografía Intravenosa (UIV). Se aprecia una litiasis que no obstruye completamente la vía de $1,5 \mathrm{~cm}$, además, los dos tercios superiores del uréter derecho están dilatados. Distalmente hay una estenosis ureteral y una segunda litiasis (Fig. 1).

En la exploración física se aprecia dolor en flanco derecho, sin masas palpables. El tacto rectal es normal. Los análisis de urgencia, tanto de sangre como de orina, no mostraron alteración alguna. La placa simple de aparato urinario objetivó una imagen radioopaca en la pelvis menor compatible con una litiasis. También se le realizó una ecografía que confirmó la existencia de una litiasis intravesical. Además se apreciaba una dilatación del remanente ureteral.

La tomografía axial computarizada (TAC) permitió definir mejor la morfología de las estructuras. Vimos que el uréter presentaba una dilación de 4 $\mathrm{cm}$ desde la vejiga hasta el hilio renal contralateral (Fig. 2).

Concluimos que la sintomatología era secundaria a la dilatación ureteral y a la presencia de la litiasis, vesical y ureteral, y se decidió practicarle una ureterectomía y una cistolitotomía por una incisión

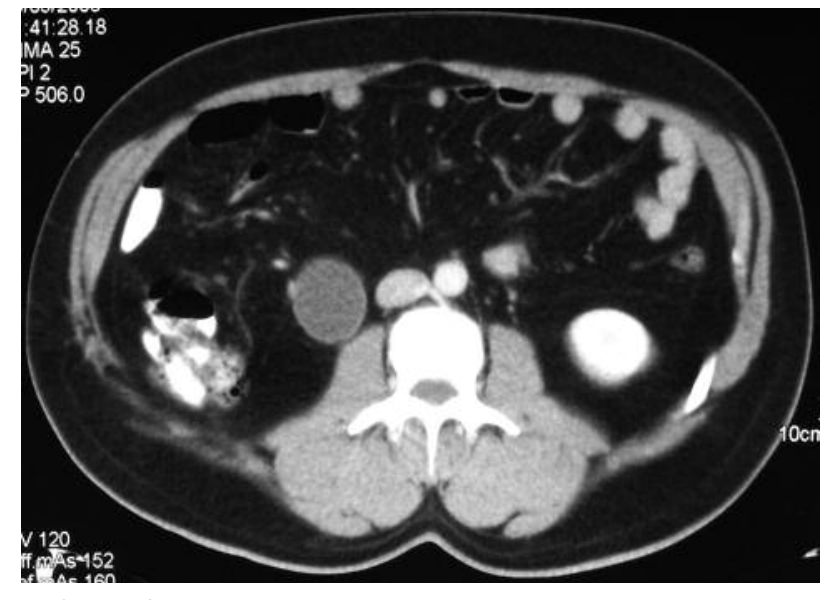

FIGURA 2

pararrectal. Durante la intervención encontramos un uréter de consistencia muy dura en su extremo distal. Se consiguió la exéresis completa aunque, debido a la adherencia a los tejidos adyacentes, se resecó también la vesícula seminal derecha. No observamos adenopatías.

El uréter medía $24 \mathrm{~cm}$ de largo por 4 de ancho, presentaba una ulceración importante, los $10 \mathrm{~cm}$ distales eran de consistencia muy dura. La luz ureteral tenía 1,5 cm. de diámetro (Fig. 3).

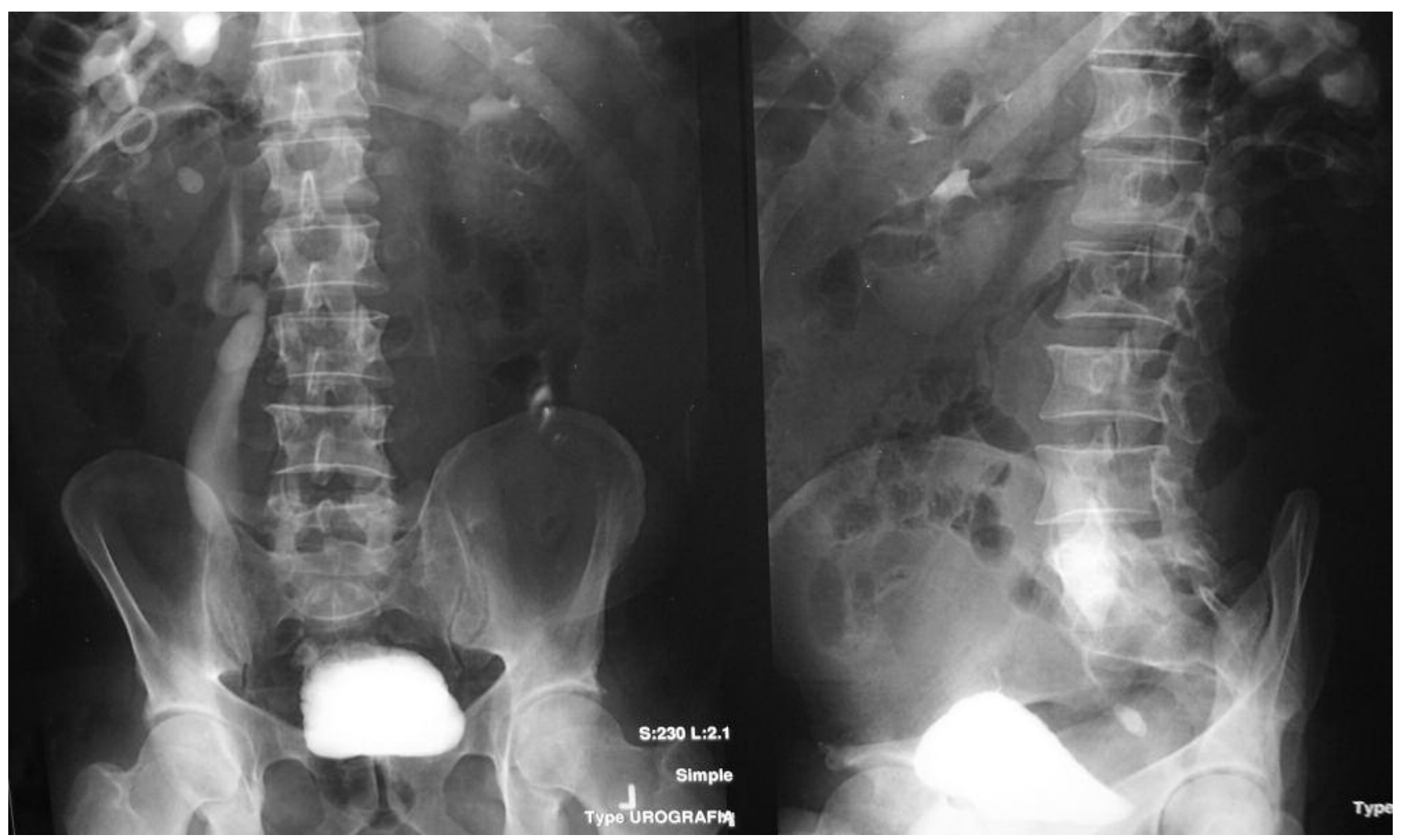

FIGURA 1 


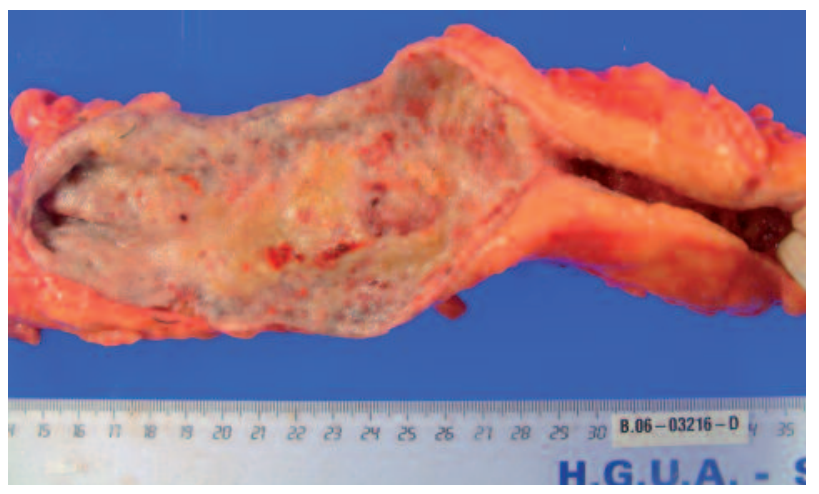

FIGURA 3

Microscópicamente se observa sustitución total del epitelio urotelial por una neoplasia maligna infiltrante (Fig. 4) de tipo adenocarcinoma intestinal moderadamente diferenciado que presenta patrones de crecimiento tubular y glandular, con pleomorfismo nuclear y marcada atipia además de numerosas figuras de mitosis. La neoplasia afecta a todo el uréter produciendo extensa infiltración en el área estenótica, infiltrando profusamente la mucosa, la muscular propia hasta el tejido adiposo periureteral, y con un crecimiento más superficial en la área dilatada. No se aprecia urotelio conservado

Todo el uréter se encontraba afectado pero la vesícula seminal estaba libre de tumor. El estudio inmunohistoquímico era intensamente positivo para citoqueratina 7 pero no expresaba citoqueratina 20 ó p53.

Los adenocarcinomas primarios de uréter son muy poco comunes, así que se solicitó una TAC toracoabdominopélvica, una colangiopancreatorresonancia, una gastroscopia y una colonoscopia para descartar un tumor primario fuera del tracto urinario, especial-

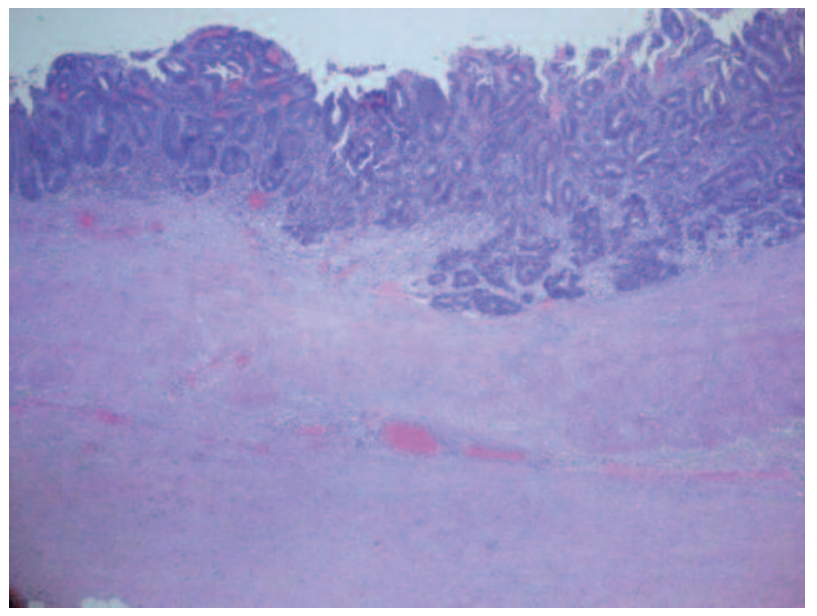

FIGURA 4 mente en el aparato digestivo. Además se solicitaron marcadores tumorales: PSA, CEA y CA 19.9. Todas las pruebas resultaron negativas a excepción de la colonoscopia en la que se resecó un pequeño pólipo. El examen anatomopatológico concluyó que se trataba de un adenoma tubular con displasia de bajo grado.

El paciente fue remitido al Servicio de Oncología donde le trató con platino a altas dosis. Tras la quimioterapia, al año y medio de la ureterectomía, presentó una metástasis única pulmonar que le fue resecada. Actualmente el paciente presenta muy buen estado general.

\section{DISCUSIÓN}

Los adenocarcinomas y los carcinomas indiferenciados del tracto urinario superior suponen, cada uno, menos de un $1 \%$ de los tumores del tracto urinario superior. El carcinoma de pelvis renal es más frecuente que el ureteral y cuando afecta al uréter lo hace con más frecuencia en el tercio distal.

Podemos sospechar un adenocarcinoma en pacientes con antecedentes de nefrectomía simple con hematuria, sindrome miccional, fiebre y/o dolor en flanco. También podemos contar con la uretrografía y la citología selectiva con catéter ureteral ${ }^{6}$. En cualquier caso el diagnóstico definitivo sólo lo dará el examen anatomopatológico tras la ureterectomía completa.

Una vez tenemos el diagnóstico anatomopatológico, debemos descartar que se trate de una metástasis originada en estómago, intestino, pulmón, páncreas, vesícula biliar o próstata ${ }^{1}$. En caso de dar con un tumor primario que pudiera dar origen a una metástasis ureteral, deben compararse ambos tejidos para cerciorarnos de que se trata del mismo tumor.

Revisamos las muestras de la pieza de la nefrectomía que se le había realizado dos años antes y no se halló ningún indicio de neoplasia. El adenoma tubular presentaba un bajo grado de displasia pero la posibilidad de que diera origen al tumor ureteral se consideró como muy baja. Sin embargo, hay un caso publicado en el que un adenoma tubular pediculado de colon presentaba un pequeño carcinoma circunscrito a su cabeza y daba origen a una invasión linfática y a metástasis a distancia ${ }^{2}$.

El manejo diagnóstico-terapéutico está resumido en la Figura 5. 


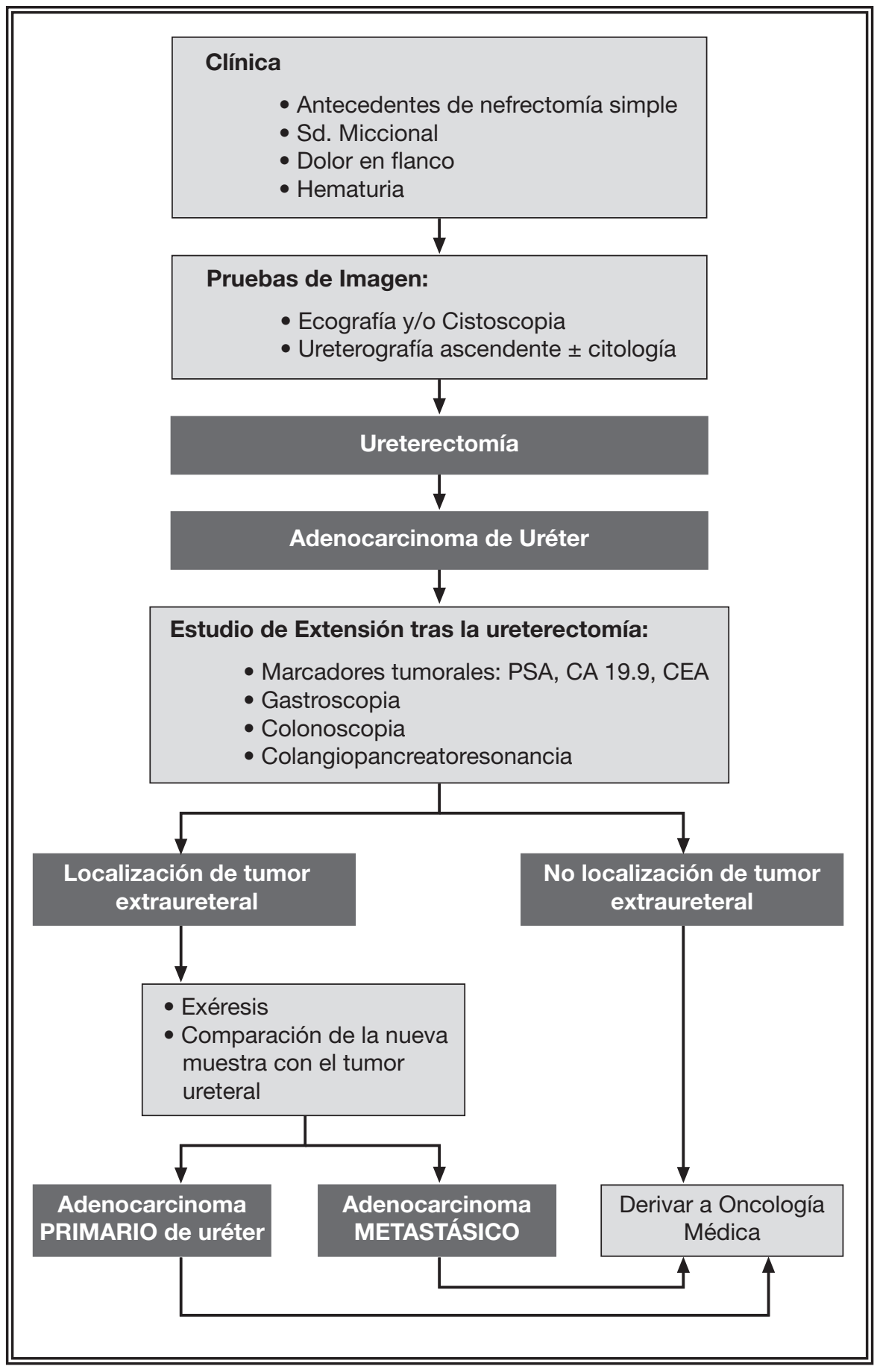

FIGURA 5. Manejo diagnóstico terapéutico de los adenocarcinomas de remanente ureteral.

La fisiopatología del adenocarcinoma de uréter se basa en una irritación prolongada de la mucosa ureteral asociada a un cuadro obstructivo e infecciones. Estos factores favorecen una metaplasia del epitelio transicional ureteral a tejido glandular ${ }^{3}$. En el 70\% de los casos comunicados existe relación con patología litiásica ${ }^{4}$.
Nuestro paciente tenía una litiasis distal a la región estenótica y no podemos descartar que se tratara del origen de la enfermedad. La litiasis ureteral pudo favorecer una reacción inflamatoria crónica en el remanente ureteral que condicionó la metaplasia de la mucosa ureteral.

También algunas anomalías congénitas, como los riñones displásicos que drenan a la vesícula seminal, pueden producir una irritación crónica urotelial ${ }^{5}$. En este caso, la vesícula seminal se resecó porque la disección fue complicada pero no presentaba ninguna anomalía anatómica.

El epitelio transicional había sido sustituido en su totalidad por el adenocarcinoma y presentaba áreas de patrón intestinal. Sin embargo estaban demasiado localizadas como para clasificarlo como adenocarcinoma de tipo intestinal.

En cuanto a las características inmunohistoquímicas, no existe un patrón de referencia debido al escaso número de casos. Algunos autores sugieren que pueden ser de utilidad, para diferenciar un adenocarcinoma primario vesical de un adenocarcinoma vesical metastásicos, los marcadores CDX-2, CK20, y CK7. En los adenocarcinomas vesicales metastásicos la CK7 sería positiva y los marcadores CDX-2 y CK20 negativos y al contrario en los primarios ${ }^{7-10}$. Nosotros hallamos una CK7 positiva típica de los tumores transicionales y no de los adenocarcinomas primarios de uréter. Pero dada la carencia de datos respecto a los adenocarcinomas ureterales no podemos sacar conclusión alguna al respecto.

El pronóstico de los pacientes con adenocarcinoma primario de uréter suele ser muy malo, algunas series sugieren que la supervivencia media es aproximadamente de 24 meses. 


\section{CONCLUSIONES}

El adenocarcinoma primario de uréter es una entidad muy poco frecuente lo que condiciona una pobre caracterización clínica y anatomopatológica. Puede presentarse como un síndrome miccional y/o hematuria en pacientes con antecedentes de nefrectomía. Ante el hallazgo de un adenocarcinoma de uréter debemos descartar siempre que se trate de un tumor metastásico. En cuanto al tratamiento parece necesaria la exéresis completa del tumor y se puede intentar el tratamiento quimioterápico adyuvante con resultados inciertos.

\section{REFERENCIAS}

1. Yui Y, Okumura S, Yoshida K, Akimoto M. Metastatic ureteral tumour from the digestive organ: a case report. Hinkokika Kiyo. 1984;30(5):691-694.

2. Stamm B, Ristivojevic B. Small pedunculated tubular adenoma of the colon with carcinoma restricted to the head, invasion of lymphatics and widespread metastases. Case report and review of the literature. Virchows Arch A Pathol Anat Histopathol. 1983;402 (1):83-89.

3. Tocchi A, Mazzoni G, Miccini M, Bettelli E, Cassini D, Brozzetti S. Primary adenocarcinoma of the ureter. Case report. G Chir. 2004;25(8-9):291-293.
4. Salm R. Combined intestinal and squamous metaplasia of the renal pelvis. J Clin Path March. 1969;22(2):187-191.

5. Brawer MK, Waisman J. Mucinous adenocarcinoma probably arising in the renal pelvis and ureter: a case report. J Urol. 1980;123(3):424-425.

6. Pollack HM, Banner MP, Popky GL. Radiologic evaluation of the ureteral stump. Radiology. 1982;144(2);225-230.

7. Haitel A, Wiener HG, Susani M. Primary adenocarcinoma of the ureter. Case report. with inmunohistochemical characterization. Pathol Res Pract. 1996;192(1):81-85.

8. Weiss MA, Mills SE. Atlas of genitourinary tract disorders, ed 1, New York: Gower. Medical Pub. 1988.

9. Litlekalsoy J, Vatne V, Hostmark JG, Laerum OD. Immunohistochemical markers in urinary bladder carcinomas from paraffin-embedded archival tissue after storage for 5-70 years. BJU Int. 2007;99(5):1013-1019.

10. Raspollini MR, Nesi G, Baroni G, Girardi LR, Taddei GL. Immunohistochemistry in the differential diagnosis between primary and secondary intestinal adenocarcinoma of the urinary bladder. Appl Immunohistochem Mol Morphol. 2005; 13 (4):358-62.

Correspondencia autor: Dr. Juan P. Caballero Romeu Servicio de Urología.

Hospital General Universitario de Alicante

Pintor Baeza, 12 - 03010 Alicante. Tel.: 965938310

E-mail autor: juanpablocaballero@gmail.com

Información artículo: Nota clínica

Trabajo recibido: septiembre 2007

Trabajo aceptado: octubre 2007 